\title{
Calculations of the pressure drop in the natural circulation boiler evaporator
}

\author{
Stawomir Grądziel ${ }^{1, *}$, Karol Majewski ${ }^{1}$ \\ ${ }^{1}$ Institute of Thermal Power Engineering, Cracow University of Technology, al. Jana Pawła II 37, 31-864 Kraków, Poland
}

\begin{abstract}
The paper presents different models used to determine pressure losses in two-phase flows: the homogeneous model, the Lockhart-Martinelli, the Friedel and the Chisholm phase-slip models and the Martinelli-Nelson graphical method. The pressure losses are calculated for the evaporator of an OP-210 boiler with the output of $210 \times 103 \mathrm{~kg} / \mathrm{h}$ operating in one of the Polish power plants. The results obtained by means of the presented models are compared to each other.
\end{abstract}

\section{Introduction}

Two-phase flows occur in many industrial processes. In the power sector, the change in the state of aggregation takes place primarily in the power boiler evaporator and in the condenser. The appearance of the steam-water mixture involves a change in the thermal and flow properties. The thermal and flow conditions change considerably in the boiler evaporator, where the content of steam in the mixture varies with the amount of heat supplied along the medium flow path. The appearance of the steam-water mixture results in phenomena which cannot be observed in single-phase flows. The pressure drop should be determined taking account of interphase forces, the shape and size of bubbles, the velocity of individual phases (the split ratio) and many other factors $[1,3,4]$.

The pressure drop can be calculated using many mathematical models. In literature, the steam-water mixture is treated as homogenous or it is considered under the phase-slip model assumptions. There are also models using graphical relations intended for the pressure drop determination. The paper presents results of the pressure drop in the OP-210 boiler evaporator determined by means of the homogeneous model, the split-phase models (the Lockhart-Martinelli, the Friedel, the Chisholm model) and the Martinelli-Nelson graphical method $[1,3,4,10]$.

\section{Structure of two-phase flows in power boilers}

Most power boiler evaporators in Poland are made of smooth tubes. The installed units are usually natural circulation boilers. For them, the steam dryness factor $x$ at the risers outlet must not exceed 0.2. Due to that, the circulation multiplicity $k$ cannot be smaller than 5:

$$
\mathrm{x}=\frac{1}{\mathrm{k}}
$$

The phenomena occurring in the boiler evaporator involve supercooled boiling and fully-developed nucleate boiling. The steam-water mixture flow structures in vertical channels are shown in Fig. 1.

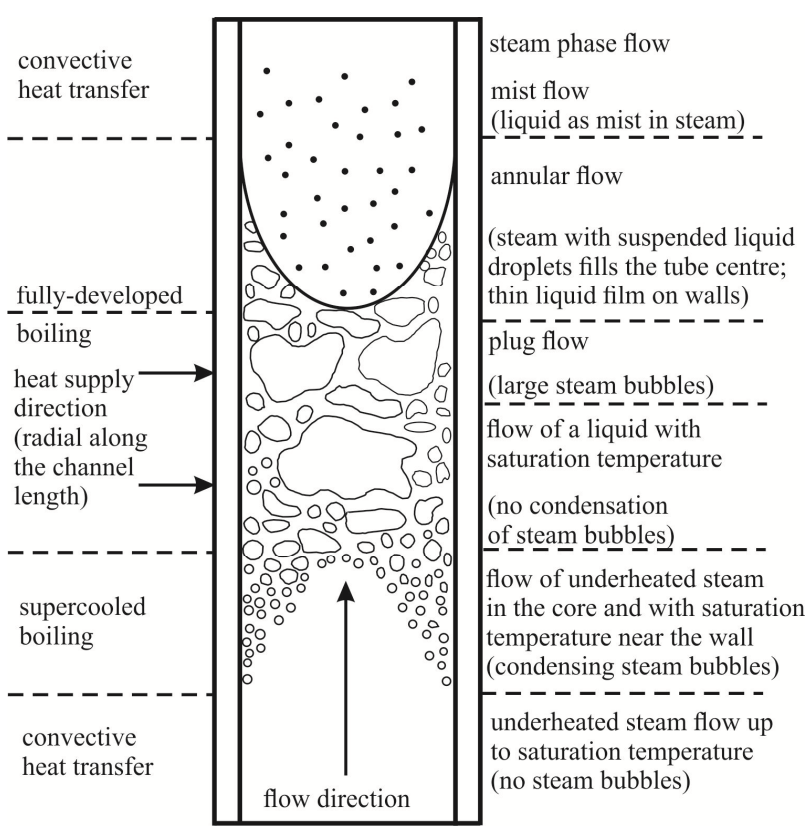

Fig. 1. Two-phase flow patterns for water-steam mixture in heated vertical channels [9].

Due to their complex flow structure, multi-phase flows are described by means of many different models

\section{Models used for the pressure drop determination}

\footnotetext{
* Corresponding author: gradziel@mech.pk.edu.pl
} 
In the general case, the total pressure drop is found from the following relation [1-7]:

$$
-\frac{d p}{d z}=-\frac{d p_{f}}{d z}-\frac{d p_{a}}{d z}-\frac{d p_{g}}{d z}
$$

where:

$\frac{d p_{f}}{d z}$ - frictional of pressure gradient, $\mathrm{Pa} / \mathrm{m}$,

$\frac{d p_{a}}{d z}$ - accelerational of pressure gradient, $\mathrm{Pa} / \mathrm{m}$,

$\frac{d p_{g}}{d z}$ - gravitational of pressure gradient, $\mathrm{Pa} / \mathrm{m}$.

In Equation (1), the pressure drops related to changes in momentum and potential energy are determined from the following relation:

$$
\begin{aligned}
& \frac{d p_{a}}{d z}=\dot{G}^{2} \frac{d}{d z}\left[\frac{(1-x)^{2}}{\rho_{L}(1-\varepsilon)}+\frac{x^{2}}{\rho_{L} \rho_{G}}\right] \\
& \frac{d p_{g}}{d z}=g\left[\rho_{G} \varepsilon-\rho_{L}(1-\varepsilon)\right] \sin \alpha
\end{aligned}
$$

where:

$\dot{G}$ - mass flux, $\mathrm{kg} /\left(\mathrm{m}^{2} \mathrm{~s}\right)$,

$x$ - dryness factor,

$\varepsilon-$ filling ratio,

$\rho_{L}, \rho_{G}-$ density of the liquid and the gaseous phase, $\mathrm{kg} / \mathrm{m}^{3}$,

$\alpha$ - tube inclination angle.

Determination of frictional pressure losses is a complex task. Therefore, many mathematical models have been developed to find the friction-related component. The mixture flow may be treated as homogeneous or as a slip flow. Using slip models involves calculating the two-phase flow multiplier, which makes it possible to find the friction-related pressure drop.

\subsection{Homogenous model}

The homogenous model application is related to the assumption that the fluid properties are averaged for the whole mixture and the gaseous and the liquid phase velocities are identical. The equality of the velocities of the two phases means that there is no slip between them. For the homogeneous model, the friction-related pressure drop is found from the following relation:

$$
\frac{d p_{f}}{d z}=\frac{f_{T P}}{d_{i n}} \frac{G^{2}}{2 \rho_{T P}}
$$

where:

$\mathrm{f}_{\mathrm{TP}}-$ two-phase friction factor,

$\dot{\mathrm{G}}$ - mass flux, $\mathrm{kg} /\left(\mathrm{m}^{2} \mathrm{~s}\right)$,

$\rho_{\text {TP }} \quad-$ two-phase density, $\mathrm{kg} / \mathrm{m}^{3}$.
The friction factor is found using classical equations, e.g. the Blasius formula, based on the Reynolds number found for averaged properties of the mixture [3, 4].

\subsection{The Lockhart-Martinelli model}

The Lockhart-Martinelli model is based on finding twophase multipliers (the Lockhart-Martinelli multipliers), which make it possible to determine the pressure gradient related to frictional losses arising during the flow of the mixture [4]:

$$
\frac{d p_{T P}}{d z}=\varphi_{L}^{2} \frac{d p_{L}}{d z}
$$

The two-phase multiplier used in equation (5) is found from the following relation:

$$
\varphi_{L}^{2}=1+\frac{C}{X}+\frac{1}{X^{2}}
$$

The Martinelli parameters $X$ and $C$ used in equation (6) are described in [4].

\subsection{The Friedel model}

The Friedel model was developed based on 25 thousand measuring points. It is used for two-phase flows in vertical and horizontal tubes. The two-phase multiplier is found from the following relation [4]:

$$
\varphi_{L O}^{2}=E+\frac{3.24 F H}{F r^{0.045} W e^{0.035}}
$$

Two-phase multiplier $\varphi_{L O}^{2}$ concerns the flow of a mixture characterized by properties related to the liquid phase properties. Coefficients E, F, H and the Froude and the Weber numbers (Fr and We, respectively) used in equation (7) are described in [4].

\subsection{The Chisholm model}

The Chisholm model is an empirical method that can be used in a wide range of the steam pressure and dryness factor values. The two-phase multiplier is found from the following formula [4]:

$$
\varphi_{L O}^{2}=1+\left(Y^{2}-1\right)\left[B x^{0.5(2-n)}(1-x)^{0.5(2-n)}+x^{2-n}\right]
$$

where: $\mathrm{Y}^{2}$ - the single-phase flow ratio between the gaseous and the liquid phase pressure gradients, B coefficient dependent on the mass flow (mass flux), $\mathrm{n}-$ exponent describing the flow resistance coefficient. For the Blasius formula $n=0.25$.

The methods of determination of parameters $\mathrm{Y}^{2}$ and $\mathrm{B}$ are described in [4].

\section{5 - The Martinelli-Nelson graphical method}


The Martinelli-Nelson method was developed on the example of flows of the steam-water mixture through horizontal ducts in the pressure range of $0.689 \div 20.7$ $\mathrm{MPa}$. The two-phase multiplier needed to establish friction-related losses is read from a chart developed based on different values of the steam pressure and dryness factor $x$ (cf. Fig. 2).

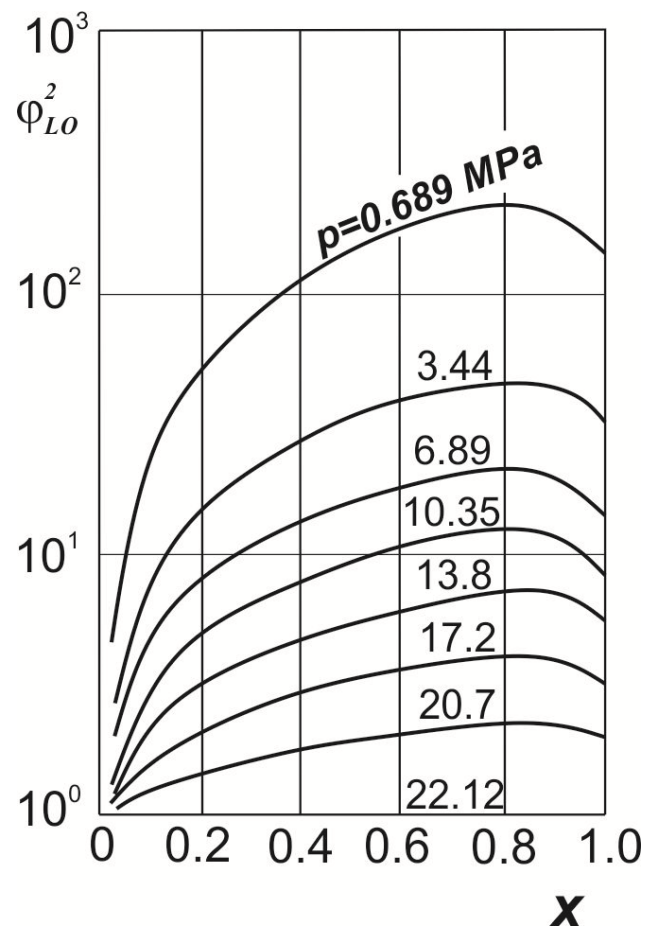

Fig. 2. Martinelli-Nelson correlation.

If the pressure value exceeds the critical point for water, the multiplier is $\varphi_{L O}^{2} \approx 1$ [11]. The two-phase multiplier read from the chart enables determination of the friction-related pressure drop by means of the following formula:

$$
\frac{d p_{f}}{d z}=\varphi_{L O}^{2} \frac{d p_{f, L O}}{d z}
$$

where:

$\frac{d p_{f, L O}}{d z}$ - frictional losses arising in the flow of a twophase mixture with properties related to the properties of the liquid phase, $\mathrm{Pa} / \mathrm{m}$.

\section{Comparison of the OP-210 boiler evaporator pressure drops determined by means of different methods}

The OP-210 boiler is a single-drum natural circulation boiler. It generates superheated steam with the pressure of $9.8 \mathrm{MPa}$ and temperature of $540^{\circ} \mathrm{C}$. The boiler nominal output totals $210 \times 103 \mathrm{~kg} / \mathrm{h}$. The pressure drop in the boiler evaporator was determined using the methodology described in [8]. The OP-210 boiler analysed circulation system, or contour, (with marked division into evaporation zones) is presented in Fig. 3.
The medium flow velocity and the degree of evaporation in the OP-210 boiler circulation contour were performed for the heat flux real values. The measurement methodology and the obtained results are presented in $[2,10]$.

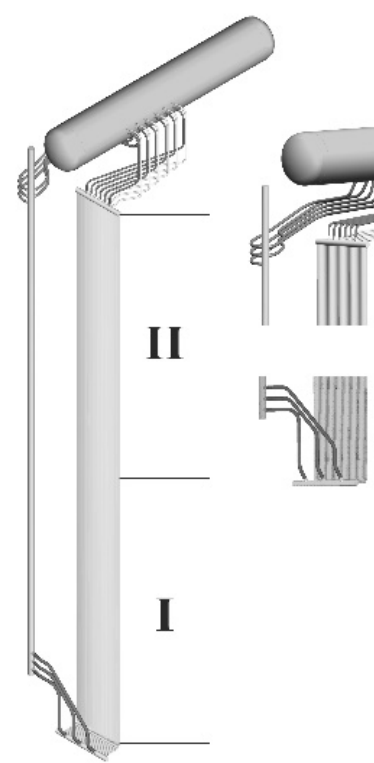

Fig. 3. Evaporator scheme of OP-210 power boiler.

Heating up to saturation parameters and partial evaporation of boiler water occur in the waterwall tubes. The calculation methodology is based on the evaporator division into a few zones [8]. Evaporation occurs in two zones so that the steam dryness factor at the outlet of the first and the second evaporation section is $x_{I}=0.01$ and $x_{2}=0.07$, respectively. A rise in the dryness factor value involves bigger friction-related pressure drops. The resistances obtained using the methodology described in [8], the homogenous model and the phase-slip models are compared in Table 1 and Fig. 4.

Table 1. Comparison of results.

\begin{tabular}{|c|c|c|c|}
\hline & & Section I & Section II \\
\hline \multicolumn{2}{|c|}{$\frac{d p_{a}}{d z}, \mathrm{~Pa} / \mathrm{m}$} & 1546 & 2399 \\
\hline \multicolumn{2}{|c|}{$\frac{d p_{g}}{d z}, \mathrm{~Pa} / \mathrm{m}$} & 6019 & 3879 \\
\hline \multirow{6}{*}{$\begin{array}{l}\frac{d p_{f}}{d z} \\
\mathrm{~Pa} / \mathrm{m}\end{array}$} & Model 1 & 142 & 208 \\
\hline & Model 2 & 174 & 260 \\
\hline & Model 3 & 318 & 968 \\
\hline & Model 4 & 211 & 375 \\
\hline & Model 5 & 194 & 348 \\
\hline & Model 6 & 110 & 396 \\
\hline $\begin{array}{l}\text { Model } 1 \\
\text { Model } 2 \\
\text { Model } 3 \\
\text { Model } 4 \\
\text { Model } 5 \\
\text { Model } 6 \\
\end{array}$ & $\begin{array}{l}\text { ature-base } \\
\text { logenous n } \\
\text { Lockhart- } \\
\text { Friedel mo } \\
\text { Chisholm } 1 \\
\text { Martinelli- }\end{array}$ & li model & \\
\hline
\end{tabular}


The calculation results indicate that the frictionrelated pressure drops take similar values for most of the models under consideration. Some differences occur for the Lockhart-Martinelli method, which was developed based on flow measurements of the air-water and air-oil mixture.

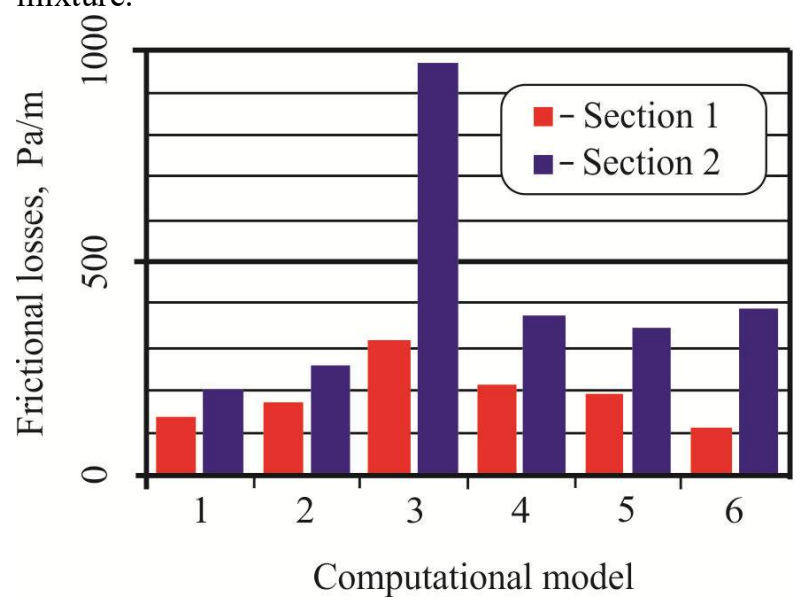

Fig. 4. Comparison of frictional losses established by means of different models: 1 - literature-based [8], 2 - homogenous model, 3 - the Lockhart-Martinelli model, 4 - the Friedel model, 5 - the Chisholm model, 6 - the Martinelli-Nelson graphical method

The obtained results indicate that frictional losses contribute to the total pressure drop only slightly. This is related to the small value of the steam dryness factor $\mathrm{x}$ and to the boiler evaporator vertical structure. Due to that, it is the changes in the mixture potential energy and momentum that have the dominant impact on the level of the total pressure drop.

\section{Conclusions}

It follows from the calculations that a rise in the gaseous phase content in the flowing mixture involves an increase in friction-related flow resistances. The applied models differ in their approach to the two-phase flow multiplier determination, which results in some differences. However, in the flow case under analysis, the content of the gaseous phase in the mixture is small and, therefore, the discrepancies between individual models are slight. The share of frictional pressure losses in the overall pressure drop is relatively small. For this reason, it does not really matter which model is selected to determine frictionrelated pressure losses - the selection has little impact on the total pressure drop in the circulation system of natural circulation boilers. The changes in the mixture potential energy and momentum have the dominant effect on the total pressure drop.

\section{References}

1. Dziubiński M., Prywer J., Two-phase fluid mechanics (WNT, Warszawa, 2009)

2. Grądziel S., Modelling of thermal and flow phenomena occurring in evaporator of boiler with natural circulation (Wydawnictwo Politechniki Krakowskiej, Kraków, 2012)

3. Hetsroni G., Handbook of Multiphase System, (Hemisphere Publishing Corporation, Washington, 1982)

4. Hewitt G., Shires G., Bott T., Process heat transfer (CRC Press, Begell House, 1994)

5. P. Ocłoń, M. Nowak, S. Łopata, J. of Therm. Sci., 23, 2, pp. 177-186 (2014)

6. P. Ocłoń, M. Nowak, K. Majewski, AIP Conference Proceedings, 1558, pp. 2419-2422 (2013)

7. P. Ocłoń, M. Nowak, B. Węglowski, T. Nabagło, P. Cisek, M. Jaremkiewicz, K. Majewski, J. of Appl. Comp. Sci., 22, 1, pp. 111-135 (2014)

8. Kuznetsov N.W., Nitor W.W., Dubovski I.E., Karasina E.S., Thermal Calculations of Steam Boilers. Standard Method (Energy, Moscow, 1973)

9. Taler J., Thermal and flow processes in large steam boilers. Modelling and monitoring (PWN, Warszawa, 2011)

10. Zima W., Grądziel S., Simulation of transient processesin heating surfaces of power boilers (LAP LAMBERT Academic Publishing,Germany, 2013)

11. R.C.Martinelli, D.B.Nelson, Trans. Amer. Soc. Mech. Engrs., 70, 6, pp. 695-702 (1948) 